\title{
mHealth in Mental Healthcare: the Application of Mobile Head-mounted Displays
}

\author{
Collin Turbyne $^{1}$ (D) $\cdot$ Pelle de Koning ${ }^{1} \cdot$ Damiaan Denys $^{1}$
}

Published online: 25 August 2017

(C) Springer International Publishing AG 2017

Research in head-mounted display (HMD) technology is presently receiving a great deal of attention in both commercial and academic settings. However, by no means is it a new technology (see Sutherland 1968). Only recently have researchers established terminology for this type of technology, which reflects the form of content being generated. There are three different types of media that are used to create a virtual environment (VE). Virtual reality (VR) is a stereoscopic, computer-generated VE that an individual can explore from any perspective. This medium is an absolute augmentation since the construction of the environment is entirely virtual, which by extension means the user's vision is completely separated from their natural environment. Augmented reality (AR) enables an individual to experience stereoscopic, computer-generated images that are overlaid into their natural environment. This medium is a more partial augmentation based on the ability to either add features to the user's natural environment or separate the user from certain features of their natural environment. Mixed reality is a combination of both VR and AR. The presentation of this content fluctuates between both forms so as to weave an experience that consists of both elements.

Clinicians are increasingly utilizing desktop-based HMD technologies as a means for advancing assessment techniques in mental health. Virtual environments enable clinicians to recreate realistic situations or environments that would otherwise be very difficult or impossible to present within traditional clinical settings. Moreover, the nature of VEs is that they are

Collin Turbyne

c.a.turbyne@amc.uva.nl

1 Department of Psychiatry, Academic Medical Center, Amsterdam, The Netherlands controlled and uniform structures, which allows for consistent experiences over time. Therefore, VEs allow clinicians to more objectively and reliably evaluate their patients' symptoms and the progression of those symptoms across multiple treatment sessions. Altogether, the clinical relevance of this technology is to help reduce subjective evaluations of psychiatric symptoms that diminish diagnostic objectivity. Despite the advantages provided by this technology, clinicians are still hesitant to adopt it mainly due to the financial implications of necessarily purchasing specialized, costly equipment as well as the high expenses involved with developing and maintaining an application.

The necessity for being able to accurately interpret symptoms is apparent; however, the potential solutions for doing so remain unclear. This issue is deeply complicated by contextual factors, such as how these symptoms are expressed during outpatient treatment as opposed to at home situations. Clinicians often remedy this situation by performing house visits, as this permits them to see the natural manifestations of their patients' symptoms. Despite its efficacy, these symptom assessments are infrequently performed due to logistical (e.g., travel distance) and financial (e.g., time consuming) restrictions. On this basis, we argue that researchers should shift their attention from desktop-based HMDs to smartphonebased HMDs to power media with clinical content for virtual exposure therapies in both clinical and non-clinical settings.

Smartphone-based HMDs are functionally similar to desktop-based HMDs; however, they are self-contained units. This means that they have all of the necessary hardware components built into them which enables their portability. These devices can be differentiated from mobile HMDs in that while mobile HMDs are also self-contained units, there is dedicated hardware powering these devices rather than a smartphone. Even though some researchers have recently begun to adopt smartphone-based HMD solutions, research outside of case 
study designs is virtually nonexistent. Furthermore, while clinically diverse, the amount of case studies within any particular disorder is limited. We believe that by placing a larger focus on developing clinical content on smartphone-based HMDs over other HMD platforms, the clinical adoption rate of HMD technology in general will be significantly accelerated and expanded by providing a more cost-effective model for developing clinical applications.

Total sales of smartphones to end-users by the end of 2016 have been estimated to be around 1.49 billon units globally (Gartner Says Worldwide 2017). By leveraging the ubiquity of smartphones, clinicians can substantially reduce the costs of adopting HMD technology from a hardware perspective by relying on the patients to use their own devices to run an application. From a software perspective, developers can further reduce costs by developing an application on a multiplatform game engine, such as Unity $3 \mathrm{D}$ or Unreal Engine. This grants developers the ability to deploy their application on any mobile operating system (e.g., Android, iOS, and Windows). Moreover, the accessibility of these game engines allows developers to rapidly prototype and test their application, which in turn further reduces major developmental expenditures.

The clinical relevance of smartphone-based HMDs is that clinicians can either personally or remotely administer an invariable exposure both inside and outside of traditional outpatient settings. By running an identical application across an unrestricted range of testing environments, clinicians can more comprehensively examine contextual effects on an individualto-individual basis. While certain practical, clinical issues arise in the case of remotely administering an exposure, smartphones are already well equipped to begin circumventing these concerns. Some of the different hardware components contained within smartphones can be integrated into an exposure to enhance its utility. GPS can be used to enforce a clinician's discretion by permitting the application to run only in select, preapproved environments, so that controlled conditions can be better maintained. The ability to restrict the use of an application based on location helps to avoid situations that either have no clear clinical benefits or pose unnecessary risks to the patient. Another useful sensor is Bluetooth, which can be used to incorporate peripheral devices that monitor and record physiological measurements. Aside from being able to quantify treatment effects, these devices can also be used as a safety switch that prevents the continued use of an application if the user's physiological state falls outside of a predefined range. Furthermore, by deploying an exposure on a smartphone, clinicians can better safeguard against unauthorized access to the sensitive, health related, data that is collected and transmitted during an exposure session. This can be accomplished by requiring additional login procedures via SMS or biometric scan (i.e., two-step login verification).

Removing the obstacles that preclude the ability to efficiently administer symptom assessments is an everexpanding area of interest in mental healthcare. Based on these considerations, developing media that are supported on smartphone-based HMDs should be a priority for clinicians that will use or are currently using HMD technology. In the Department of Psychiatry at the Academic Medical Center (Amsterdam, The Netherlands), we are currently developing a smartphone-based HMD virtual exposure therapy for individuals with contamination-based obsessive-compulsive disorder. This application is a markerless, GPS-based, AR solution that enables us to render computer-generated stains (i.e., dirt, fecal matter, blood, and other bodily fluids) onto a variety of real-world objects (i.e., door handles, light switches, telephones, and toilets) located around the hospital. The participants are led to different, pre-approved, locations around the hospital through the use of AR navigational paths to different scenes. Each scene consists of one of the computer-generated stains augmented over one of the real world objects. Meanwhile, the clinician is able to monitor the participant remotely on their computer by mirroring the participant's screen and accessing the phone's microphone. This application enables the participants to realistically explore different scenarios that they may encounter in real-life situations by using a controlled VE without necessitating the direct presence of a clinician. The data we collect from these exposures consists of self-report questionnaires, which participants answer after exploring each scene, and physiological recordings from our ambulatory monitoring system that we fit to each participant before they begin their exposure session. While evidence for the use of smartphone-based HMDs is still being built up, future directions for research should include (a) validating assessment tools, (b) validating peripheral devices, and (c) developing approaches that are mediated by an artificially intelligent assistant.

\section{References}

Gartner Says Worldwide Sales of Smartphones Grew 7 Percent in the Fourth Quarter of 2016. (2017) Retrieved from http://www.gartner. com/newsroom/id/3609817.

Sutherland, I. E. (1968). A head-mounted three dimensional display. Proceedings of the December 9-11, 1968, Fall Joint Computer Conference, Part I on - AFIPS '68 (Fall, Part I). https://doi.org/10. 1145/1476589.1476686. 